\title{
Winter ambient training conditions are associated with increased bronchial hyperreactivity and with shifts in serum innate immunity proteins in young competitive speed skaters
}

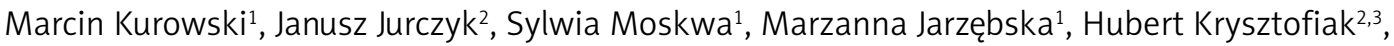 \\ Marek L. Kowalski ${ }^{1}$
}

1Department of Immunology, Rheumatology and Allergy, Healthy Ageing Research
Centre, Medical University of Lodz, Lodz, Poland
${ }^{2}$ National Centre for Sports Medicine (COMS), Warsaw, Poland
${ }^{3}$ Mossakowski Medical Research Centre, Polish Academy of Sciences, Warsaw, Poland

Submitted: 9 May 2016

Accepted: 26 September 2016

Arch Med Sci 2018; 14, 1: 60-68

DOI: https://doi.org/10.5114/aoms.2017.69438

Copyright (c) 2017 Termedia \& Banach

\section{Abstract}

Introduction: Regular training modulates airway inflammation and modifies susceptibility to respiratory infections. The impact of exercise and ambient conditions on airway hyperreactivity and innate immunity has not been well studied. We aimed to assess exercise-related symptoms, lung function, airway hyperresponsiveness and innate immunity proteins in relation to meteorological conditions and exercise load in competitive athletes.

Material and methods: Thirty-six speed skaters were assessed during winter (WTP) and summer (STP) periods. The control group comprised 22 non-exercising subjects. An allergy questionnaire for athletes (AQUA) and IPAQ (International Physical Activity Questionnaire) were used to assess symptoms and exercise. Meteorological parameters were acquired from World Meteorological Organization resources. Serum innate immunity proteins were measured by ELISA.

Results: Exercise-associated respiratory symptoms were reported by $79.4 \%$ of skaters. Despite similar exercise load and lung parameters during both periods, positive methacholine challenge was more frequent during winter $(p=0.04)$. Heat shock protein HSPA1 and IL-1RA were significantly decreased during STP compared to WTP and controls. During WTP, IL-1RA was elevated in skaters reporting exercise-induced symptoms $(p=0.007)$. sCD14 was elevated in athletes versus controls in both periods $(p<0.05)$. HSPA1 was significantly higher in WTP compared to STP irrespective of presence of respiratory tract infections (RTIS). IL-1RA in WTP was elevated versus STP $(p=0.004)$ only in RTI-negative athletes. Serum IL-1RA negatively correlated with most meteorological parameters during WTP.

Conclusions: Ambient training conditions, but not training load, influence bronchial hyperreactivity and the innate immune response in competitive athletes assessed during winter. The protective effect of regular exercise against respiratory infections is associated with a shift in serum innate immunity proteins.

Key words: interleukin-1 receptor antagonist protein, meteorological factors, exercise training, bronchial hyperreactivity, heat shock protein HSPA1, SCD14.

\author{
Corresponding author: \\ Prof. Marek L. Kowalski \\ Department of \\ Immunology, \\ Rheumatology \\ and Allergy \\ Healthy Ageing \\ Research Centre \\ Medical University \\ of Lodz \\ Pomorska 251 bud. C-5 \\ 92-213 Lodz, Poland \\ Phone: +48 426757309 \\ E-mail: marek.kowalski@csk. \\ umed.lodz.pl
}




\section{Introduction}

Regularly performed strenuous high-load exercise contributes to deterioration of immunocompetence and may increase systemic inflammation and susceptibility to infection $[1,2]$. Despite observations associating respiratory tract infections (RTIs) with acute heavy-load exercise bouts, evidence remains inconclusive and other causes of exercise-associated airway discomfort are considered [3]. The common belief is that moderate regular exercise enhances immune functions and decreases RTI frequency and, contrarily, that intensive training impairs anti-infectious defense [3-5]. Recent evidence suggests that physically inactive subjects might be more prone to RTIs immediately after exercise due to exercise-associated modification of innate antimicrobial defense [6]. The relation between exercise intensity and susceptibility to infections has been presented as a J-shaped curve model [5]. Increased RTI frequency in athletes remains in direct association with higher training intensity [7-9]. Exercise-associated modifications of the anti-microbial response are held responsible for this phenomenon [10] but microbial pathogen could be identified in less than one third of RTI cases in athletes [11]. These findings gave rise to the hypothesis that infection-like symptoms reflect non-infectious inflammatory stimuli. This may be due to loss of control of chronic low-grade airway inflammation [3]. In this context, moderate regular exercise would down-regulate inflammation and prevent infections. Data regarding the inflammatory response during and after acute exercise are available [7, 12,13 ] but less is known about the influence of exercise on the innate immune response over a longer period. Additionally, ambient conditions influence the immune response and inflammation $[14,15]$, but few studies have been performed regarding exercise, respiratory infections and innate immunity $[15,16]$.

In this study we aimed to assess selected parameters of innate immunity as related to environmental conditions and exercise load during two training periods: winter and summer. Innate immunity proteins selected for assessment included:

- cytokines with strong pro-inflammatory properties: tumor necrosis factor- $\alpha$ (TNF- $\alpha$ ), interleukin 6 (IL-6), and IL-1 $\beta$,

- IL-10 and interleukin 1 receptor antagonist (IL-1RA), which are regarded as having antiinflammatory and immunomodulatory properties and exercise-associated decreased concentrations of which were described in infection-prone subjects [7],

- soluble form of bacterial lipopolysaccharide (LPS) receptor CD14 (sCD14), which is a marker of monocyte activation,
- heat shock protein A1 (HSPA1), which is a "danger signal" for innate immunity mechanisms associated with natural killer (NK) cell and monocyte activation.

Exercise-associated respiratory symptoms, lung function and bronchial hyperreactivity were also assessed.

\section{Material and methods}

The study group consisted of 36 subjects (19 males) aged 11-21 (median age 15 years) performing speed skating at the competitive level for at least three seasons. To avoid possible selection bias, we invited all athletes from the local speed skating section without any incentive for those self-suspecting asthma or allergy. Four athletes $(11.8 \%)$ were previously diagnosed with allergic disease: asthma $(n=1)$, conjunctivitis $(n=1)$, food allergy $(n=1)$ and rhinitis $(n=2)$. Symptoms of rhinitis were reported by $26.5 \%$ and conjunctivitis by $14.7 \%$ of athletes. The control group consisted of 22 healthy, non-allergic, non-asthmatic and non-smoking medical students not performing physical exercise regularly.

Athletes were assessed at two time-points: the first between the beginning of January and the end of March (winter training period - WTP), and the second between the beginning of May and the end of July (summer training period - STP). The WTP was characterized by high training intensity in unfavorable winter conditions and frequent participation in tournaments at outdoor ice rinks. During STP athletes performed regular training consisting mainly of roller skating on the outdoor facility. Overall exercise load during 1 and 4 weeks before assessment was similar in both periods (Table I). No abstention from exercise was required before assessment. Usually, previous training activity took place in the afternoon before assessment.

Healthy controls were assessed once during late summer, and assessment of physical activity was based on subjects' declarations.

Data concerning atmospheric conditions were obtained from the Weather Information Service (available at http://www.ogimet.com/gsynres.phtml.en accessed 5 October 2015). The service was searched for data gathered at the World Meteorological Organization (WMO) station 12466 in Tomaszów Mazowiecki, Poland.

The following weather parameters were included in the analyses: mean daily temperature on assessment (TO); mean daily temperature during 7 preceding days (T-7); mean daily temperature during 28 preceding days ( $\mathrm{T}-28)$; lowest temperature on assessment day; median lowest $\mathrm{T}$-7; median lowest T-28; relative humidity on assessment; mean relative humidity over preced- 
Table I. Lung function parameters, percentage positive methacholine challenge tests and physical activity load in speed skaters during winter and summer training seasons

\begin{tabular}{|c|c|c|c|}
\hline Parameter & Winter training period & Summer training period & $P$-value \\
\hline $\mathrm{FEV}_{1}[\%$ predicted $]$ & 99 (94-109) & $97(93-112)$ & $>0.05^{a}$ \\
\hline $\mathrm{FEV}_{1} / \mathrm{FVC}$ & $0.83(0.79-0.87)$ & $0.83(0.78-0.87)$ & $>0.05^{a}$ \\
\hline MEF25-75 [\%predicted] & $105(95-123)$ & $99(91-121)$ & $>0.05^{a}$ \\
\hline IPAQ 1 week [MET-h/week] & $7553(3360-10050)$ & $7553(4857-11150)$ & $>0.05^{a}$ \\
\hline Mean IPAQ 4 weeks [MET-h/week] & 7518 (4059-9996) & $8176(4340-10740)$ & $>0.05^{a}$ \\
\hline
\end{tabular}

ing 7 days; dew point temperature on assessment day; and mean dew point over preceding 7 days.

Data regarding history of allergy and exercise-associated symptoms were acquired from the Allergy Questionnaire in Athletes (AQUA) [17] completed by $34(94.4 \%)$ athletes entering the study. Additionally, author-designed specific questions regarding respiratory discomfort associated with skating were given separately.

Athletes completed the Polish version of the International Physical Activity Questionnaire (IPAQ) providing information on exercise load over the preceding week [18]. To acquire more detailed information on training-specific exercise load, data on vigorous exercise were extracted from IPAQ and included in the analysis as a separate parameter. On the assessment day subjects filled in 4 IPAQ sheets providing retrospective data on exercise in the 4 preceding weeks. This was done in order to check for fluctuations in the training regime directly before assessment.

Spirometry was performed using Lungtest 1000 equipment (MES, Krakow, Poland). Non-specific bronchial challenge with methacholine was carried out in accordance with the guidelines issued by ATS [19] using an inhalation system for allergen provocation (ISPA) compatible with the Lungtest 1000 spirometer (MES, Krakow, Poland).

Skin prick tests (SPTs) were performed with a panel of eighteen seasonal and perennial allergens (Allergopharma, Reinbek bei Hamburg, Germany).

Blood was drawn after 8-hour fasting into Monovette tubes for serum (Sarstedt, Landskrona, Sweden). Samples were left to clot at room temperature for $30 \mathrm{~min}$ and then centrifuged at 2,000 $g$ for $10 \mathrm{~min}$. Serum was frozen at $-80^{\circ} \mathrm{C}$ for further analyses. Levels of TNF- $\alpha$, IL- 6, IL-10, IL-1RA, IL-1 $\beta$, sCD14 and HSPA1 were measured by ELISA using commercially available kits from Gen-Probe Diaclone SAS, Besançon, France (TNF- $\alpha$, IL-1 $\beta$, IL-6, IL-10, sCD14) and USCN Life Science Inc., Wuhan, People's Republic of China (IL-1RA, HSPA1), according to the manufacturers' instructions.
The study protocol was approved by the Bioethics Commission of the Medical University of Lodz. Subjects or legal guardians gave written informed consent for participation, study procedures and biological material storage.

\section{Statistical analysis}

Statistical analyses were carried out using Statistica 12 (StatSoft, Tulsa, OK, USA) and GraphPad Prism 4.00 (GraphPad Software, San Diego, CA, USA). Data were tested for a normal distribution using the Shapiro-Wilk normality test. Regarding continuous variables following a normal distribution, the paired or non-paired $t$ test was used for comparisons between observations. For non-normally distributed data, the Mann-Whitney $U$ test or Wilcoxon signed rank test was used. Univariate associations between variables were evaluated using Spearman rank correlation. To identify the best set of variables contributing to changes in continuous dependent variables, multiple regression analyses were performed. Stepwise forward regressions were used (a variable with an $F$ value of 4.00 or higher was retained in the model). To identify the best set of variables that contributed to changes in dichotomous variables, logistic analyses were performed. A $p$-value less than 0.05 was considered statistically significant.

\section{Results}

Exercise-associated respiratory symptoms were reported by $79.4 \%(n=27)$ of skaters ("EIB group"). Among them, cough and/or chest tightness were present in $77.8 \%(n=21)$, dyspnea in $55.6 \%$ $(n=15)$ and wheezing in $48.1 \%(n=15)$ of athletes.

Symptomatic skaters most frequently (88.9\%) described their symptoms as "not occurring during every exercise training". One (3.7\%) skater had a single incident of EIB symptoms, while $2(7.4 \%)$ skaters stated that they had respiratory symptoms upon every exercise session. Respiratory symptoms usually started within the first $10 \mathrm{~min}$ post-exercise (81.5\%) and lasted up to $30 \mathrm{~min}$ (median time: $10 \mathrm{~min}$ ). In $7.4 \%$ of athletes 
symptoms started during exercise and in $11.1 \%$ more than 10 min after exercise. Only $6(22.2 \%)$ symptomatic skaters believed that exercise-induced dyspnea impaired their performance, and in 3 skaters training exercise had ever been interrupted by respiratory symptoms. Only 1 athlete ever used a bronchodilator on demand because of exercise-induced symptoms.

Pulmonary function parameters in all athletes were within the normal range and did not differ between study periods (Table I).

The methacholine challenge test performed in both training periods in 21 out of 36 athletes was positive in $42.9 \%(n=9)$ during WTP and in $19.1 \%$ $(n=4)$ skaters during STP $(p=0.04)$. Methacholine PD20 did not differ significantly between WTP and STP (0.38 mg (0.08-1.80) vs. 0.57 mg (0.06-5.35), geometric means $(95 \% \mathrm{Cl}))$. From among four athletes with methacholine challenge positive in both periods, in three PD20 remained at a similar level during both assessments, whereas a notable bronchial hyperresponsiveness (BHR) decrease was observed in one athlete (PD20 change from $1.51 \mathrm{mg}$ in WTP to $2.94 \mathrm{mg}$ in STP).

The SPTs were performed in 32 athletes and atopy was determined in 11 (34.4\%) athletes.

In summer, HSPA1 and IL-1RA levels were significantly lower in athletes than in $\mathrm{HC}$ in the same season (Figures 1 A, B). Athletes had significantly higher SCD14 levels in both seasons as compared to HC (Figure $1 \mathrm{C}$ ) For serum HSPA1 and IL-1RA but not for SCD14, significant decreases in STP were observed in all athletes (Figure 2). Serum concentrations of TNF- $\alpha, \mathrm{IL}-6, \mathrm{IL}-10$ and IL- $1 \beta$ were detectable only in single subjects.

Serum IL-1RA levels in WTP were significantly higher in skaters reporting exercise-related respiratory symptoms compared to asymptomatic athletes (25.1 (15.6-107.5) vs. 12.6 (11.95-20.7) pg/ml, respectively, $p=0.007$, medians (interquartile ranges)), whereas HSPA1 (1.04 (0.87-1.2) vs. 0.99 (0.75-1.63) $\mathrm{ng} / \mathrm{ml}, p=0.98)$ and sCD14 (181.0 (162.9-217.8) vs. 193.8 (161.5-210.9) ng/ml, $p=0.85)$ did not differ between symptomatic and asymptomatic subjects. During STP serum levels of all proteins did not differ between these groups (data not shown).

Eight (22.2\%) skaters self-reported frequent RTI symptoms in the AQUA questionnaire. Serum HSPA1 levels were significantly higher in WTP versus STP ( $p=0.002$ and $p=0.0001$, respectively) both in RTI-positive and RTI-negative athletes (Figure $3 \mathrm{~A}$ ). The mean serum IL-1RA level in WTP was significantly higher than in STP $(p=0.004)$ only in RTI-negative subjects (Figure $3 \mathrm{~B}$ ). No differences in serum SCD14 between training periods were found in the context of self-reported RTIs.
A

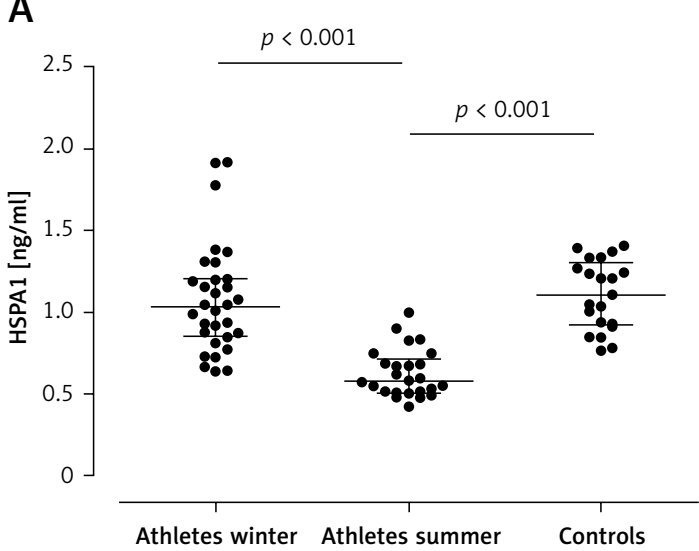

C

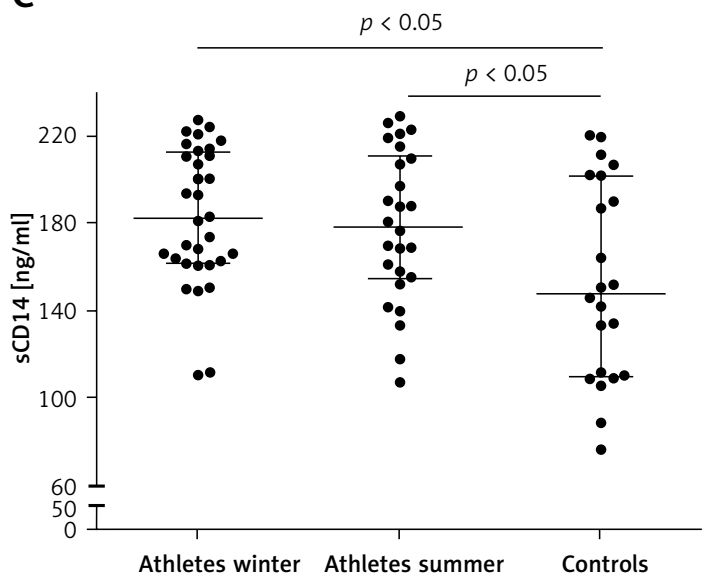

B

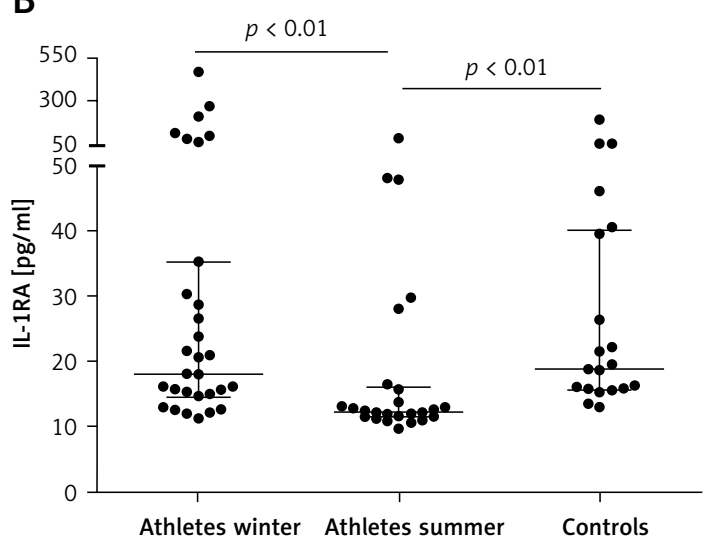

Figure 1. Serum concentrations of heat shock protein A1 (A), interleukin 1 receptor antagonist (B) and soluble CD14 (C) in athletes during summer and winter training periods and in control subjects 
A

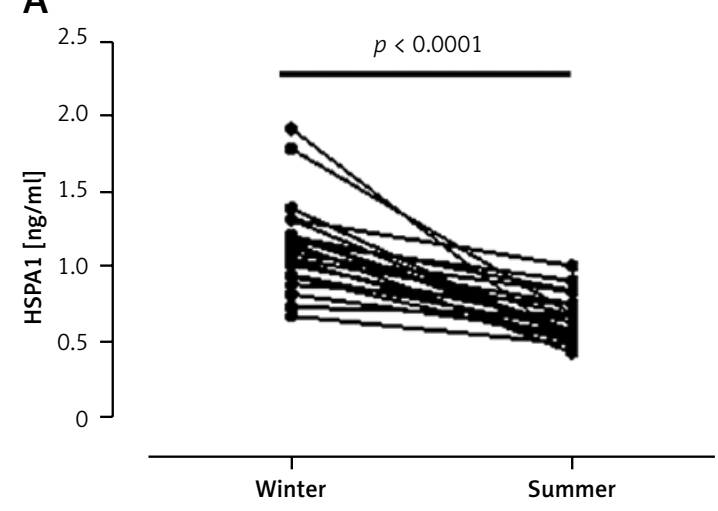

C

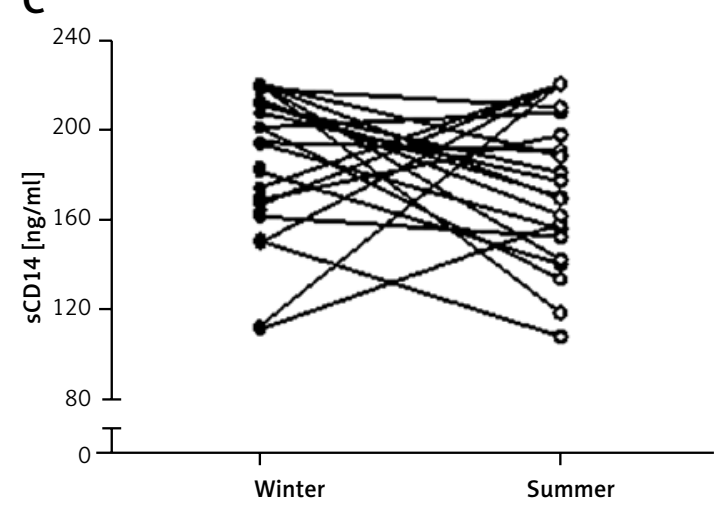

B

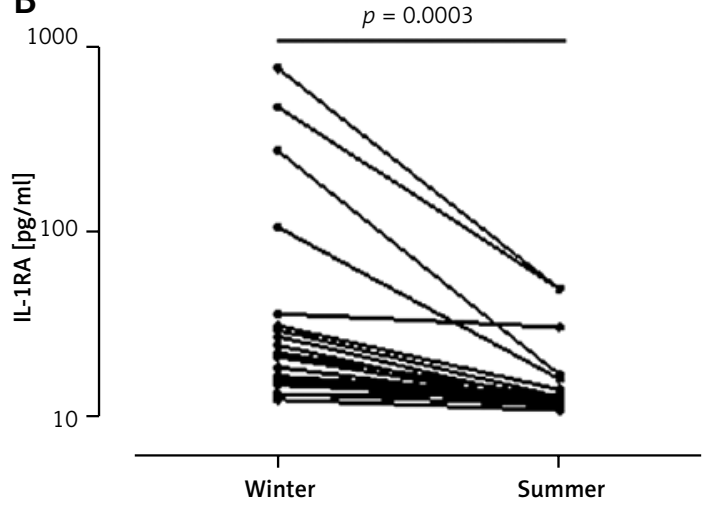

Figure 2. Individual changes in serum levels of heat shock protein A1 (A), interleukin 1 receptor antagonist (log scale) (B) and soluble CD14 (C) between winter and summer training periods

A

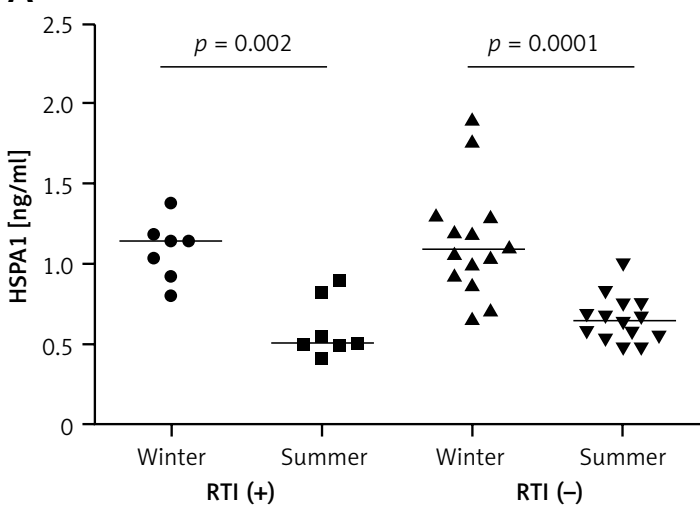

C

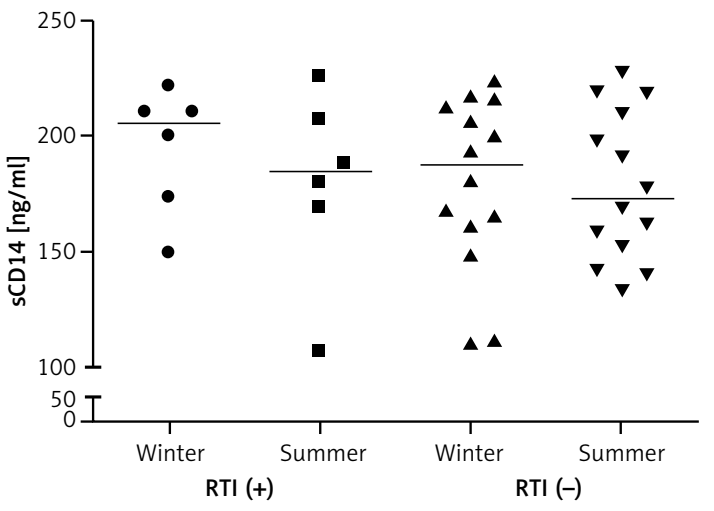

B

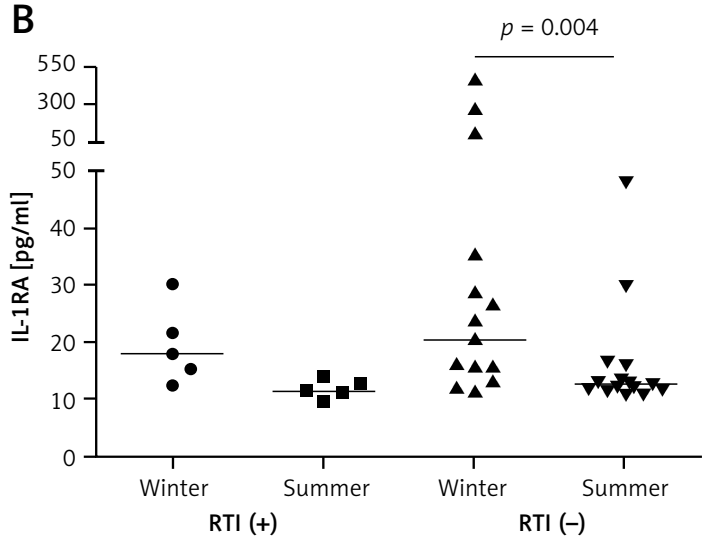

Figure 3. Serum concentrations of heat shock protein A1 (A), interleukin 1 receptor antagonist (B) and soluble CD14 (C) in athletes reporting and not reporting frequent respiratory infections (RTI (+) and $\mathrm{RTI}(-)$, respectively) during winter and summer training periods 
During WTP significant negative correlations were observed between serum IL-1RA and various measurements of ambient temperature: TO (average and lowest); T-7 (average and median lowest), average T-28 and dew point temperature on assessment day.

Significant positive correlations were observed between IL-1RA and relative air humidity on the day of assessment and during 7 days before (Table II).

A statistically significant regression model could be built with regard to log values of serum IL-1RA with relative humidity on assessment day as the sole retained variable. Such a model explained $16.6 \%$ of serum IL-1RA variability (coefficient of determination, $\left.R^{2}=0.16594\right)$, and an increase of relative humidity by 1 percent-point resulted in a mean $0.048 \%$ increase in serum IL-1RA level (regression coefficient, $b=0.048 ; p=0.02$ ).

In STP, significant negative correlations were observed between serum SCD14 and T-7 and T-28 (average and median highest) as well as average dew point temperature over 1 week before assessment. Similar correlations were observed for serum HSPA1 and T-28 (Table III).

A statistically significant regression model could be built with regard to serum concentrations of SCD14, HSPA1 and IL-1RA during STP.

Regarding SCD14, the variable to be retained in the constructed model was the median highest T-7. Such a model explained $41.4 \%$ of sCD14 serum concentration variability $\left(R^{2}=0.41414\right)$, and an increase of the median highest temperature during the preceding 7 days by 1 degree resulted in mean fall of serum SCD14 by $5.36 \mathrm{ng} / \mathrm{ml}(b=-5.36 ; p<0.003)$.

For HSPA1, the retained variable was mean T-28. This model explained $32.1 \%$ of HSPA1 serum concentration variability $\left(R^{2}=0.32072\right)$, and an increase of mean temperature during the preceding 4 weeks by 1 degree resulted in a mean fall of serum HSPA1 by $0.027 \mathrm{ng} / \mathrm{ml}(b=-0.027 ; p<0.02)$.

Regarding IL-1RA, the constructed model, with mean 4-week exercise load as the retained variable, explained $26.7 \%$ of IL-1RA serum concentration variability $\left(R^{2}=0.26673\right)$ and an increase of mean 4-week exercise load by 1 MET-min/week resulted in a mean increase of serum IL-1RA by $0.0018 \mathrm{pg} / \mathrm{ml}(b=0.00177 ; p<0.03)$.

Correlations of IL-1RA level with exercise load in athletes were observed in both periods. Serum IL-1RA was positively correlated with total and vigorous exercise load during the preceding 7 and 28 days in WTP and during the preceding 28 days in STP (Tables II, III).

In $\mathrm{HC}$, weak but significant negative correlations were observed between serum IL-1RA and average $(R=-0.44, p<0.05)$ and lowest T0 $(R=$ $-0.45, p<0.04)$ and between serum HSPA1 and highest TO $(R=-0.43, p=0.049)$.

\section{Discussion}

In this study we assessed competitive skaters during two training periods contrasting with re-

Table II. Correlations of serum SCD14, HSPA1 and IL-1RA with weather parameters and activity load in the winter training period (WTP)

\begin{tabular}{|c|c|c|c|c|c|c|}
\hline \multirow[t]{2}{*}{ Variable } & \multicolumn{2}{|c|}{ sCD14 } & \multicolumn{2}{|c|}{ HSPA1 } & \multicolumn{2}{|c|}{ IL-1RA } \\
\hline & $R$ & $p$ & $R$ & $p$ & $R$ & $p$ \\
\hline TO & 0.02 & 0.89 & -0.09 & 0.64 & -0.51 & 0.003 \\
\hline $\mathrm{T}-7$ & 0.10 & 0.58 & -0.04 & 0.82 & -0.56 & $<0.001$ \\
\hline T-28 & 0.11 & 0.56 & 0.07 & 0.70 & -0.49 & 0.004 \\
\hline Lowest temp. day 0 & 0.12 & 0.52 & -0.007 & 0.97 & -0.22 & 0.23 \\
\hline Median lowest temp. week 1 & 0.23 & 0.20 & 0.08 & 0.67 & -0.38 & 0.03 \\
\hline Median lowest temp. week 4 & 0.17 & 0.36 & 0.11 & 0.54 & -0.21 & 0.26 \\
\hline Relative humidity day 0 & 0.19 & 0.29 & -0.02 & 0.93 & 0.55 & 0.001 \\
\hline Mean relative humidity week 1 & 0.02 & 0.92 & 0.02 & 0.92 & 0.43 & $<0.02$ \\
\hline Dew point day 0 & 0.09 & 0.61 & -0.12 & 0.51 & -0.39 & 0.03 \\
\hline Dew point week 1 & 0.07 & 0.69 & -0.02 & 0.93 & -0.33 & 0.06 \\
\hline IPAQ & -0.14 & 0.44 & 0.05 & 0.80 & 0.46 & $<0.01$ \\
\hline Mean IPAQ week 4 & 0.03 & 0.89 & -0.02 & 0.92 & 0.35 & 0.055 \\
\hline IPAQ (vigorous) & -0.23 & 0.20 & -0.09 & 0.64 & 0.51 & 0.003 \\
\hline Mean IPAQ (vigorous) week 4 & -0.05 & 0.80 & -0.12 & 0.53 & 0.48 & 0.006 \\
\hline
\end{tabular}

Significant correlations marked in bold print. $R$-Spearman rank correlation coefficient. 
Table III. Correlations of serum sCD14, HSPA1 and IL-1RA with weather parameters and activity load in the summer training period (STP)

\begin{tabular}{|c|c|c|c|c|c|c|}
\hline \multirow[t]{2}{*}{ Variable } & \multicolumn{2}{|c|}{ sCD14 } & \multicolumn{2}{|c|}{ HSPA1 } & \multicolumn{2}{|c|}{ IL-1RA } \\
\hline & $R$ & $p$ & $R$ & $p$ & $R$ & $p$ \\
\hline T0 & -0.25 & 0.22 & 0.10 & 0.62 & -0.06 & 0.78 \\
\hline $\mathrm{T}-7$ & -0.65 & 0.0003 & -0.15 & 0.46 & 0.004 & 0.98 \\
\hline $\mathrm{T}-28$ & -0.45 & 0.02 & -0.58 & 0.002 & -0.005 & 0.98 \\
\hline Highest temp. day 0 & -0.20 & 0.31 & 0.11 & 0.61 & -0.03 & 0.88 \\
\hline Median highest temp. week 1 & -0.67 & 0.0002 & -0.16 & 0.44 & -0.02 & 0.92 \\
\hline Median highest temp. week 4 & -0.56 & 0.003 & -0.51 & 0.008 & -0.03 & 0.89 \\
\hline Relative humidity day 0 & 0.15 & 0.46 & 0.14 & 0.48 & 0.07 & 0.72 \\
\hline Mean relative humidity week 1 & -0.06 & 0.76 & 0.30 & 0.13 & -0.01 & 0.97 \\
\hline Dew point day 0 & -0.19 & 0.33 & 0.29 & 0.14 & 0.09 & 0.66 \\
\hline Dew point week 1 & -0.54 & $<0.005$ & -0.15 & 0.47 & 0.19 & 0.36 \\
\hline IPAQ & -0.11 & 0.61 & -0.19 & 0.35 & 0.3 & 0.13 \\
\hline Mean IPAQ week 4 & -0.12 & 0.55 & -0.14 & 0.51 & 0.39 & 0.046 \\
\hline IPAQ (vigorous) & 0.36 & 0.07 & -0.07 & 0.72 & 0.24 & 0.23 \\
\hline Mean IPAQ (vigorous) week 4 & 0.01 & 0.94 & -0.17 & 0.39 & 0.49 & 0.01 \\
\hline
\end{tabular}

Significant correlations marked in bold print. $R$-Spearman rank correlation coefficient.

spect to ambient conditions, but involving a similar exercise load. A very high proportion of athletes (almost 80\%) reported exercise-associated respiratory symptoms. Although basic respiratory function in athletes did not differ between seasons, during winter almost twice as many athletes demonstrated BHR to methacholine. We suppose that high prevalence of non-specific bronchial responsiveness during winter is secondary to airway inflammation and does not indicate presence of persistent asthma [20]. Airway inflammation during the winter season in speed skaters may result from exposure to unfavorable ambient conditions leading to airway epithelium damage [21]. Aeroallergens and pollutants also contribute to airway inflammation in outdoor exercisers [22, 23]. Our population was relatively homogeneous in terms of residence area; therefore, the influence of pollutants on airway inflammation can be regarded as evenly affecting all participants. Aeroallergens may have influenced airway inflammation in atopic subjects, but the most spectacular shifts in serum proteins and correlations with ambient parameters were observed during winter, when few inhalant allergens are present in the atmosphere.

Exercise performed in unfavorable external conditions may enhance airway inflammation. In subjects exercising in warm and humid conditions, changes in serum pro- and anti-inflammatory cytokines were described [24]. Our results confirm the role of ambient conditions in exercise-associated inflammation. We found strong evidence that serum IL-1RA reflects exposure to low air temperature during outdoor speed skating and that the influence of cold air on inflammation is not limited to acute bouts of exercise but extends over a longer period of training. This observation is further supported by the positive correlation between serum IL-1RA and exercise load in WTP. Considering the fact that no correlation between air temperature and IL-1RA was found outside the WTP, we may hypothesize that IL-1RA is partly specific in reflecting cold air-induced inflammatory changes. It should be emphasized that the exercise load was similar in both training seasons. This fact, along with increased serum IL-1RA in RTI-resistant athletes during winter, may suggest that unfavorable ambient conditions and not exercise load - constitute the primary factor stimulating airway inflammation and influencing infection susceptibility in outdoor athletes. The stimulatory effect exerted on IL-1RA release by chronic exercise per se is equally present, since the correlation of IL-1RA and exercise load was observed in summer. However, the role of winter conditions in enhancing and perpetuating airway inflammation is, in our opinion, not negligible.

Studies regarding inflammatory cytokines in regular exercisers bring varying results. Henson et al. [25] found no significant difference between adolescent tennis players and controls in terms of 
serum/plasma IL-1RA and respiratory infections over 2.5 months. During the training season, no significant modifications in lymphocyte counts but a significant decrease in intracellular IL-2 and IL-4 production was described in Italian footballers [26]. Contrarily, a Portuguese study of kayakers in the off training season revealed lower plasma IL-1 $\beta$, IL-18, IFN- $\gamma$ and IL-1RA, confirming the beneficial long-standing anti-inflammatory effect of regular intensive exercise [27]. Anti-inflammatory and protective effects of exercise against stimuli potentially harmful for the respiratory system were also described in animal models $[28,29]$. Release of IL-1RA upon acute bouts of exercise has been reported $[7,30]$. Through its biological activity IL-1RA contributes to anti-inflammatory effects of moderate exercise [31]. Our findings regarding increased IL-1RA levels in RTI-resistant athletes during WTP are concordant with the anti-inflammatory spectrum of IL-1RA activity. Previously, decreased IL-1RA levels after exercise in illness-prone subjects were reported by Cox et al. [7]. In our study, subjects considered less RTI-prone had significantly higher serum IL-1RA during training in unfavorable conditions. Resistance to infection in subjects at risk may therefore be associated with altered regulation of inflammation [32]. In this regard, our results are similar to those published by Cox et al. [7] although their observations cover the first $24 \mathrm{~h}$ after exercise.

An acute exercise stimulus increases circulating heat-shock proteins (HSPs) [33]. Our results suggest that also chronic exercise, when performed in unfavorable ambient conditions, may increase serum circulating HSPs. Resting serum HSPA1 is usually lower in trained versus untrained individuals [33]. Regarding athlete groups in the present study, we may assume that summer serum HSPA1 reflects resting HSPA1 status. Elevated serum HSPA1 during the training season would therefore reflect the response to chronic exercise aiming to boost the immune response.

Our results also suggest that elevated sCD14 reflects constant pro-inflammatory stimulation during the training period, irrespective of ambient conditions, and is probably the sequel to exercise-induced release of pro-inflammatory cytokines. In contrast to HSPA1 and IL-1RA, we have not ascertained any association of serum SCD14 with RTIs in athletes. This is in contrast to findings associating elevated SCD14 with recurrent infections due to possible modifications of the response to LPS [34]. Changes in SCD14 attributable to regular exercise certainly require further clarification.

We cannot provide a plausible explanation for the undetectable serum levels of TNF- $\alpha$, IL-6, IL-10 and IL-1 $\beta$ found in most subjects. Since these proteins were detected in single subjects, we can probably exclude technical faults and the influence of external factors on the final result. It is possible that in our populations the baseline levels were below detection limits and would have been ascertainable only in a period directly following training exercise, whereas our subjects were not assessed in relation to the training session.

Evaluation of control subjects at a single timepoint limits the possibility of extending our results to the non-exercising population and may be considered as hindering their interpretation. Assessment of non-exercisers during winter and summer might have provided some insight into the influence of ambient conditions on innate immunity parameters in the general population. We observed that serum HSPA1 and IL-1RA levels in healthy controls were similar to those observed in skaters during the winter training season. This suggests that both innate immunity boosting (HSPA1) and anti-inflammatory stimuli are present, independently of exercise stimulation. Studies specifically designed for investigating the reasons behind these phenomena are therefore needed.

In conclusion, we have documented that ambient training conditions, but not training load, may influence bronchial hyperreactivity and the innate immune response in competitive athletes. Furthermore, training at a competitive level influences serum concentrations of selected innate immunity proteins, and ambient conditions constitute an important factor influencing the innate immune response and potentially altering susceptibility to infection in competitive outdoor winter athletes. Association of meteorological parameters with innate immune response proteins was noted both in athletes and controls; however, this effect was significantly more pronounced in athletes. Finally, our data suggest that the protective effect of regular exercise against respiratory infections may result, in part, from shifts in serum innate immunity protein levels, observed during periods of intensive exercise training.

\section{Acknowledgments}

This study was financed by Ministry of Science and Higher Education of the Republic of Poland grant no. 5981/B/P01/2011/40.

The authors wish to acknowledge the contribution of Ms. Dorota Żaromińska, RN, to questionnaire data collection and clinical assessment of subjects, as well as that of Mr. Bartosz Grancow, MSc to statistical analysis.

\section{Conflict of interest}

The authors declare no conflict of interest. 


\section{References}

1. Gleeson M, Bishop N. URI in athletes: are mucosal immunity and cytokine responses key risk factors? Exerc Sport Sci Rev 2013; 41: 148-53.

2. Pokrywka A, Zembron-Lacny A, Baldy-Chudzik K, Orysiak J, Sitkowski D, Banach M. The influence of hypoxic physical activity on cfDNA as a new marker of vascular inflammation. Arch Med Sci 2015; 11: 1156-63.

3. Walsh N, Gleeson M, Shephard R, et al. Position statement. Part one: immune function and exercise. Exerc Immunol Rev 2011; 17: 6-63.

4. Gleeson M, Bishop N, Oliveira M, Tauler P. Influence of training load on upper respiratory tract infection incidence and antigen-stimulated cytokine production. Scand J Med Sci Sports 2013; 23: 451-7.

5. Nieman D. Immune response to heavy exertion. J Appl Physiol 1997; 82: 1385-94.

6. Kiwata J, Anouseyan R, Desharnais R, Cornwell A, Khodiguian N, Porter E. Effects of aerobic exercise on lipid-effector molecules of the innate immune response. Med Sci Sports Exerc 2014; 46: 506-12.

7. Cox A, Pyne D, Saunders P, Callister R, Gleeson M. Cytokine responses to treadmill running in healthy and illness-prone athletes. Med Sci Sports Exerc 2007; 39: 1918-26.

8. Nieman D, Henson D, Dumke C, Lind R, Shooter L, Gross S. Relationship between salivary IgA secretion and upper respiratory tract infection following a $160-\mathrm{km}$ race. J Sports Med Phys Fitness 2006; 46: 158-62.

9. Robson-Ansley P, Howatson G, Tallent J, et al. Prevalence of allergy and upper respiratory tract symptoms in runners of London Marathon. Med Sci Sports Exerc 2012; 44: 999-1004.

10. Nieman D. Exercise, upper respiratory tract infection, and the immune system. Med Sci Sports Exerc 1994; 26: $128-39$

11. Spence L, Brown W, Pyne D, et al. Incidence, etiology, and symptomatology of upper respiratory illness in elite athletes. Med Sci Sports Exerc 2007; 39: 577-86.

12. Seys SF, Hox V, Van Gerven L, et al. Damage-associated molecular pattern and innate cytokine release in the airways of competitive swimmers. Allergy 2015; 70: 187-94.

13. Krzemiński K, Buraczewska M, Miśkiewicz Z, et al. Effect of ultra-endurance exercise on left ventricular performance and plasma cytokines in healthy trained men. Biol Sport 2016; 33: 63-9.

14. Kim K, Suzuki K, Peake J, et al. Physiological and leukocyte subset responses to exercise and cold exposure in cold-acclimatized skaters. Biol Sport 2014; 31: 39-48.

15. Niess A, Fehrenbach E, Lehmann R, et al. Impact of elevated ambient temperatures on the acute immune response to intensive endurance exercise. Eur J Appl Physiol 2003; 89: 344-51.

16. Bonini M, Gramiccioni C, Fioretti D, et al. Asthma, allergy and the Olympics: a 12-year survey in elite athletes. Curr Opin Allergy Clin Immunol 2015; 15: 184-92.

17. Bonini M, Braido F, Baiardini I, et al. AQUA: Allergy Questionnaire for Athletes. Development and validation. Med Sci Sports Exerc 2009; 41: 1034-41.

18. Craig C, Marshall A, Sjöström M, et al. International physical activity questionnaire: 12-country reliability and validity international physical activity questionnaire: 12 -country reliability and validity. Med Sci Sports Exerc 2003; 35: 1381-95.

19. American Thoracic Society. Guidelines for methacholine and exercise challenge testing - 1999. Am J Respir Crit Care Med 2000; 161: 309-29.
20. Anderson S, Kippelen P. Exercise-induced bronchoconstriction: pathogenesis. Curr Allergy Asthma Rep 2005 5: $116-22$.

21. Seys SF, Daenen M, Dilissen E, et al. Effects of high altitude and cold air exposure on airway inflammation in patients with asthma. Thorax 2013; 68: 906-13.

22. Rundell KW, Sue-Chu M. Air quality and exercise-induced bronchoconstriction in elite athletes. Immuno Allergy Clin North Am 2013; 33: 409-21.

23. Helenius I, Tikkanen H, Haahtela T. Occurrence of exercise induced bronchospasm in elite runners: dependence on atopy and exposure to cold air and pollen. Br J Sports Med 1998; 32: 125-9.

24. Hailes WS, Slivka D, Cuddy J, Ruby BC. Human plasma inflammatory response during 5 days of exercise training in the heat. J Therm Biol 2011; 36: 277-82.

25. Henson D, Nieman D, Kernodle M, Sonnenfeld G, Morton D, Thompson M. Immune function in adolescent tennis athletes and controls. Sports Med Training Rehab 2001; 10: 235-46.

26. Del Giacco SR, Scorcu M, Argiolas F, Firinu D, Del Giacco GS Exercise training, lymphocyte subsets and their cytokines production: experience of an Italian professional football team and their impact on allergy. BioMed Res Int 2014; 2014: 429248.

27. Borges G, Rama L, Pedreiro S, et al. Differences in plasma cytokine levels between elite kayakers and nonathletes. Biomed Res Int 2013; 2013: 370354.

28. Ramos D, Olivo C, Quirino Santos Lopes F, et al. Low-intensity swimming training partially inhibits lipopolysaccharide-induced acute lung injury. Med Sci Sports Exerc 2010; 42: 113-9.

29. Vieira R, Toledo A, Silva L, et al. Anti-inflammatory effects of aerobic exercise in mice exposed to air pollution. Med Sci Sports Exerc 2012; 44: 1227-34.

30. Sugama K, Suzuki K, Yoshitani K, Shiraishi K, Kometani T. Urinary excretion of cytokines versus their plasma levels after endurance exercise. Exerc Immunol Rev 2013; 19: $29-48$.

31. Gleeson M, Bishop N, Stensel D, Lindley M, Mastana S, Nimmo $M$. The anti-inflammatory effects of exercise: mechanisms and implications for the prevention and treatment of disease. Nat Rev Immunol 2011; 11: 607-15.

32. Bermon S. Airway inflammation and upper respiratory tract infection in athletes: is there a link? Exerc Immunol Rev 2007; 13: 6-14.

33. Fehrenbach E, Niess A, Voelker K, Northoff H, Mooren F. Exercise intensity and duration affect blood soluble HSP72. Int J Sports Med 2005; 26: 552-7.

34. Lødrup Carlsen K, Granum B. Soluble CD14: role in atopic disease and recurrent infections, including otitis media. Curr Allergy Asthma Rep 2007; 7: 436-43. 